\begin{abstract}
ID: 42
\end{abstract}
Poster(Competing)

\title{
Erosive Tooth Wear: Knowledge Of Diagnosis And Treatment Strategies Among Private Dental Practitioners In Kuantan, Pahang
}

Noorhazayti Ab. Halim ${ }^{\text {a }}$ | Nur Atiyah Fakhri ${ }^{\text {b }}$ | Nur Izzati Aisyah Azhar ${ }^{\text {b }}$

${ }^{a}$ Department of Paediatric Dentistry, Orthodontics and Dental Public Health, Kulliyyah of Dentistry, International Islamic University Malaysia | ${ }^{b}$ Kulliyyah of Dentistry, International Islamic University Malaysia

Introduction: Awareness of erosive tooth wear (ETW) by the public is still low and most dental practitioners overlooked the very early stages dismissing minor tooth surface loss of erosive tooth wear. Therefore, this study aimed to assess knowledge of diagnosis and treatment strategies of ETW among private dental practitioners (PDP) in Kuantan. Materials and Methods: A crosssectional study was conducted in Kuantan private clinics involving PDP and general dental practitioners who performed locum by using convenience sampling. A self-administered validated questionnaire was distributed and descriptive analysis was performed. Results: Overall response rate was $75 \%$. Results indicated that majority of participants $(79.2 \%)$ received formal education about ETW. About half of participants further learned through continuous dental education. All participants agreed that carbonated drink was the cause of ETW and majority of them reported that gastro-esophageal reflux diseases and pickles also contributed to ETW. Regarding ETW documentation, majority of participants (79\%) preferred case notes. Nevertheless, $40.9 \%$ and $36.4 \%$ record at tooth surface level and individual level respectively. In term of specific scoring in assessing ETW, 79\% of them did not know about it. Most of participants prefer giving oral hygiene instruction and diet counseling as preventive measures; however usage of fluorides was not prioritized. $77 \%$ of participants never referred case to dental specialist and prefer to treat patient by themselves. Conclusion(s): Knowledge of ETW among recruited PDP is adequate however they did not use any specific indices for ETW scoring and treatment strategies were insufficient.

KEYWORDS: erosive tooth wear (ETW), private dental practitioners (PDP), knowledge, indices, treatment strategies 